\title{
On effect of resource constraints on optimal distribution of commodities
}

\author{
Mikhail SEVODIN \\ Faculty of Applied Mathematics and Mechanics \\ Perm National Research Polytechnic University \\ Perm, Russia \\ m.sevodin@mail.ru
}

\author{
Vladimir PERVADCHUK \\ Faculty of Applied Mathematics and Mechanics \\ Perm National Research Polytechnic University \\ Perm, Russia \\ pervadchuk@mail.ru
}

\author{
Tatiana OSECHKINA \\ Faculty of Applied Mathematics and Mechanics \\ Perm National Research Polytechnic University \\ Perm, Russia \\ mathschool_pstu@mail.ru
}

\begin{abstract}
The article investigates a closed exchange economy model. It considers situations where constraints are imposed on the volumes of purchase and sale by economic agents. At the same time, it allows a certain range for price variability. Such approaches to studying economic processes are motivated by production, economical and political reasons, forming the socalled rationing schemes. Typically, and in this work in particular, rationing schemes, applied under natural conditions, allow allocating a subset of resources and guaranteeing a commodity distribution that is not "too bad." In this article, both resource and price constraints are cone-shaped, which allows applying them to growing economies. The authors establish the existence of a rationed equilibrium under these circumstances. Unlike conventional rationing schemes, the authors also consider situations where certain consumer preferences are non-convex. The preference convexity condition is replaced with a weaker condition of convexity within the directional cone. At the same time, the proof of existence of a rationed equilibrium does not impose any significant additional constraints on the consumer.
\end{abstract}

Keywords- exchange economy, rationed equilibrium, constraints, preferences

\section{INTRODUCTION}

It is known [1-4] that non-price equilibrium concepts allow a greater freedom of choice at the cost of a lower overall system efficiency. This applies to markets where for some reason prices do not change fast enough to balance the supply and demand. In such markets, price games give way to some form of resource rationing (funding), which leads to a relatively optimal distribution of goods - although each of the economy's participants would prefer to spend the available money differently from the prescribed manner. The economy, based on such principles, was first analyzed in [5], [6]. Descriptions of some similar economies can be found in [1-6]. At the moment, the rationing schemes are widely used in various areas of the economy (see e.g. [7-10]). All economies described in these works feature bilateral resource constraints.
In this article, the authors investigate a situation where such constraints form a certain cone. Let us first describe an economic situation calling for such constraints.

Since demand is the basic driver of economic growth, stimulating it is a key element of an economic policy. Maintaining high consumer demand inevitably leads to a growing money supply. This in turn results in a proportional increase in the prices of products, that is, an adequate inflationary price increase. Thus, if one imposes constraints on the prices, such constraints must have a cone-like shape. Certain goods may grow or fall in prices - regardless of each other - but cannot escape the defined bounds. If such systems are managed through economic means, the planning authority will define a stimulating system of sanctions and rewards. This is how constraints are usually defined in the manufacturing industry. Just as the prices, such constraints, too, must be cone-shaped.

This article aims to prove that in such situation, an equilibrium is reached not only through price mechanisms, but also by quantitatively constraining the net trading volume by limiting the volume of purchases and sales by economic agents. The authors will use cone-shaped constraints in this work. Let us note that, unlike the conventional approach (see, e.g., [11]), the preferences can be non-convex here.

Convexity is an important topic in economics and is used in mathematical models of many economic phenomena. Attempts to give an economic explanation and justification of the convexity requirement instantly narrow down the possible applications of the models in question. This has been noted and discussed by numerous authors (see e.g. [12-15]). In particular, the convexity of consumption sets is universally used in all classical proofs of existence of a competitive equilibrium. At the same time, this excludes all economy participants who are unwilling to distribute their consumption among some goods [13]. For example, this applies to 
participants whose preference convexity properties depend on many factors such as e.g. risk attitude [14].

Many authors have highlighted the need to weaken the classical economic theory's assumptions requiring the existence of an economic equilibrium. Attempts to remove the convexity constraint have been mainly applied to very special economies. In particular, this included environments in which the market had one or infinitely many goods or consumers [16, 17], or where manufacturers strived to zero out their profit margins [13]. An overview of such research can be found in [18-20].

In the present article, the authors directly weaken the convexity requirement for a manufacturer's production set. Thus, this work also aims to prove that there are situations where the non-convexity of participants' consumption sets does not affect the existence of equilibrium distributions.

\section{MATHEMATICAL MODEL OF THE ECONOMY}

Consider an economy whose participants exchange goods so as to obtain sets thereof that meet their needs as much as possible (see, e.g., [12-14]). This economic model involves no taxes, no production, and - explicitly - no money.

Let $l$ be the number of economic goods, $m$ - the number of participants, and $x_{k} \geq 0$ - the quantitiy of the $k$-th good. This is equivalent to defining vector $x$ in $R_{+}^{l}-$ the nonnegative orthant of the space $R^{l}: x=\left(x_{1}, \ldots, x_{l}\right)$. Now, let $X^{i}\left(\subset R_{+}^{l}\right)$ be the consumption set - i.e., the set of sets of goods $x$ consumable by the $i$-th participant.

Let us fix the set of goods $\Omega \in R_{+}^{l}$ that is assumed to be universally known and to represent the economic reserves in their entirety. The complete resources $\Omega$ are distributed among all economic agents. In other words, if vector $\omega^{i} \in X^{i}$ represents the set of goods of the $i$-th participant, then $\Omega=\omega^{1}+\ldots+\omega^{m}$.

All participant exchange their commodities for the commodities of other participants. In a sense, they "pay" for the goods (acquired through the exchange) by a part of their resources. This leads to certain exchange proportions among goods. The authors will refer to these proportions as "prices". Vector $p=\left(p_{1}, \ldots, p_{l}\right)$ defines the price system, scaled according to the first good (i.e. $p_{1}=1$ ).

Let us assume that all prices are positive, i.e., that all goods are desired. Let us also assume that the prices are fixed and defined externally so that the participants act under these price conditions and do not influence them.

The authors formalize the relation between a consumer's preference and the set of consumer goods as a binary relation $\succeq$ on $R_{+}^{l}$, which is reflexive, transitive, and complete. We will only consider continuous preferences; hence, the binary relation in question is also continuous.

Let us further assume that $(i=\overline{1, m})$ :

1. The consumption set $X^{i}\left(\subset R_{+}^{l}\right)$ is closed, convex, and its elements satisfy the following condition: $x \in X^{i} \Rightarrow\{x\}+R_{+}^{l} \subset X^{i}$.

2. The preference ordering $\succeq_{i}$ on $X^{i}$ is complete, monotonic, continuous, and convex in the directional cone $R^{l} \backslash\left\{ \pm R_{+}^{l}\right\}[11] ; \quad x \geq y \Rightarrow x \succeq_{i} y ; \quad I \subset\{1,2, \ldots, n\}$ is the index set, where $x \geq y$ and $x_{j}>y_{j} \forall j \in I \Rightarrow x \succ_{i} y$.

3. Initial resource vector $\omega^{i}$ belongs to set $X^{i}$.

Let $z^{i}$ be the $i$-th participant's net trading volume: $z^{i}=x^{i}-\omega^{i}$.

Let us assume that price vector $p$ can deviate from defined $p^{0}$ by not more than $\alpha$. Let $\mu$ be the cosine of $\alpha$. One can then define the price set as

$$
P=\left\{p \in R_{+}^{l} \mid p_{1}=1, \rho\left(p, p^{0}\right) \leq 2 \sin \alpha / 2=\sqrt{2(1-\mu)}\right\}
$$

where $\rho(x, y)=\|x /\| x\|-y /\| y\|\|$.

Since no price equilibrium is expected, we set a constraint of $\delta \in[0 ; 1]$ on the volume of purchased and marketed commodities in order to balance the supply and demand. Here, $\delta$ is the cosine of $\varphi$, and $\varphi$ - is the maximum angle between $x$ and $\omega^{j} . \delta$ is non-negative as both vectors $x$ and $\omega^{j}$ lie within the first orthant.

Given price system $p \in P$ and $\delta$, one can define the consumer's budget set as

$$
B^{i}(p, \delta)=\left\{x \in X^{i} \mid\left\langle p, x-\omega^{i}\right\rangle \leq 0, \rho\left(x, \omega^{i}\right) \leq 2 \sin \varphi / 2\right\} .
$$

Initial resource vector $\omega^{i}$ belongs to $X^{i}$ and, obviously, to the budget set. Hence, the budget set is non-empty for all allowed $p$ and $\delta$.

Let us introduce multivalued correspondence $F^{i}(p, \delta)$ that maps each pair of $(p, \delta)$ to elements of $X^{i}$ for which $\left\langle p, x-\omega^{i}\right\rangle \leq 0, \rho\left(x, \omega^{i}\right) \leq 2 \sin \varphi / 2$. Then $B^{i}(p, \delta)$ is the output set of this correspondence, corresponding to $\operatorname{pair}(p, \delta)$.

Let us define this economic model as $E=\left\{\left(X^{i}, \succeq_{i}, \omega^{i}\right), p\right\}$.

One can say that the economy in question is in a rationed equilibrium, if $m$-dimensional consumption vector $\left\{x^{i}\right\}_{1}^{m}$, 
price system $p \in P$, and the rationing scheme satisfy the following conditions:

a) For all $i=\overline{1, m}, x^{i}$ has the highest preference $\succeq_{i}$ among all elements of budget set $B^{i}(p, \delta)$;

b) $\sum_{i=1}^{m}\left(x^{i}-\omega^{i}\right)=0$ (balance);

c) If $\left\langle x, \omega^{i}\right\rangle=\delta \cdot\|x\| \cdot\left\|\omega^{i}\right\|$ for some $i$, then $\left\langle p, p^{0}\right\rangle=\mu \cdot\|p\| \cdot\left\|p^{0}\right\|$.

Conditions $a$ ) and $b$ ) are known from [6] and need no further comments. According to $c$ ), the price of a constraineddemand good is maximal.

\section{EXISTENCE OF A RATIONED EQUILIBRIUM}

Now one needs to prove the following.

Lemma1. Correspondence $F^{i}(p, \delta)$ is both upper and lower hemicontinuous in each point $(p, \delta)$ with $p_{j}>0, j=\overline{1, l}$.

Proof. Let $\alpha^{i}(p)=\left\{x \mid x \in R^{l},\left\langle p \cdot x-\omega^{i}\right\rangle \leq 0\right\}$,

$\beta^{i}(\delta)=\left\{x \mid x \in R^{l},\left\langle x, \omega^{i}\right\rangle \geq \delta \cdot\|x\| \cdot\left\|\omega^{i}\right\|\right\}$.

Let us note that $\alpha^{i}(p), \beta^{i}(\delta)$ are convex correspondences. One knows [6] that $\alpha^{i}(p)$ is both upper and lower hemicontinuous in each point $p>0$.

Let us prove that $\beta^{i}(\delta)$ is also both upper and lower hemicontinuous. Correspondence $\beta^{i}(\delta)$ is defined on set $R$ and applies for $X^{i} \subset R^{l}$. Initial resource vector $\omega^{i}$ belongs to $X^{i}$ and, obviously, to the output set of correspondence $\beta^{i}(\delta)$. Hence, the output set of the correspondence is non-empty for all $\delta$.

Let $\quad \delta_{n} \rightarrow \delta_{0}, x^{n} \rightarrow x^{0}, x^{n} \in \beta^{i}\left(\delta_{n}\right)$.

Then $\left\langle x^{n}, \omega^{i}\right\rangle \geq \delta_{n} \cdot\left\|x^{n}\right\| \cdot\left\|\omega^{i}\right\|$ for any $n$. Thus, through passage to the limit, $\left\langle x^{0}, \omega^{i}\right\rangle \geq \delta_{0} \cdot\left\|x^{0}\right\| \cdot\left\|\omega^{i}\right\|$. Hence, $x_{0} \in \beta^{i}\left(\delta_{0}\right)$, i.e. $\beta^{i}(\delta)$ is upper hemicontinuous.

To prove lower hemicontinuity, let us assume that $\delta^{s} \rightarrow \delta^{0}, x^{0} \in \beta^{i}\left(\delta^{0}\right)$. The interior of set $\beta^{i}(\delta)$ is nonempty. Hence, there is sequence $x^{s} \rightarrow x^{0}, x^{s} \in \beta^{i}\left(\delta^{s}\right)$ at any $s$. Thus, correspondence $\beta^{i}(\delta)$ is lower hemicontinuous for all $\delta$.
Correspondence $F^{i}(p, \delta)$ is defined on set $R_{+}^{l} \times R$ and applies for $X^{i} \subset R^{l}$. Initial resource vector $\omega^{i}$ belongs to $X^{i}$ and, obviously, to the output set of correspondence $F^{i}(p, \delta)$. Hence, set $B^{i}(p, \delta)$ is non-empty for any $\delta$.

The upper hemicontinuity of correspondence $F^{i}(p, \delta)$ follows from $B^{i}(p, \delta)=\alpha^{i}(p) \cap \beta^{i}(\delta)$. To prove lower hemicontinuity of $F^{i}(p, \delta)$, let us assume that there is sequence $\left(p^{s}, \delta^{s}\right)$ converging to $\left(p^{s 0}, \delta^{s 0}\right)$, where $p_{j}^{s 0}>0, j=\overline{1, l}$. Let $x^{0} \in B^{i}\left(p^{s 0}, \delta^{s 0}\right)$.

Since $\beta^{i}(\delta)$ is both upper and lower hemicontinuous, there is sequence $\left\{\hat{x}^{s}\right\}: \hat{x}^{s} \in \beta^{i}\left(p^{s}, \delta^{s}\right)$, where $\hat{x}^{s} \rightarrow x^{0}$.

If $x^{0}$ is an interior point of $B^{i}\left(p^{s 0}, \delta^{s 0}\right)$, there is $s^{\prime}$ such that, for all $s \geq s^{\prime}, \quad \hat{x}^{s} \in \alpha^{i}\left(p^{s}, \delta^{s}\right)$. Indeed, if $\left\langle p^{s 0}, x^{0}-\omega^{i}\right\rangle<0$, then $\left\langle p^{s}, \hat{x}^{s}-\omega^{i}\right\rangle<0, s \geq s^{\prime}$.

Now let us assume that $x^{0}$ is a boundary point of $B^{i}\left(p^{s 0}, \delta^{s 0}\right)$, i.e. $\left\langle p^{s 0}, x^{0}-\omega^{\dot{ }}\right\rangle=0$. Then there is $\bar{x} \in \alpha^{i}\left(p^{s_{0}}\right) \quad$ such that $\quad\left\langle p^{s 0}, \bar{x}-\omega^{i}\right\rangle<0 \quad$ and $\bar{x} \in \beta^{i}\left(\delta^{s_{0}}\right),\left\langle p^{s}, \bar{x}-\omega^{i}\right\rangle<0, s \geq s^{\prime}$.

Let us define sequence $\left\{x^{s}\right\}$ such that $x^{s}=\left\langle\lambda^{s}, \hat{x}^{s}\right\rangle+\left\langle 1-\lambda^{s}, \bar{x}\right\rangle$ and $\left\langle p^{s}, x^{s}-\omega^{i}\right\rangle=0$, for all $s$ such that $\hat{x}^{s} \notin \alpha^{i}\left(p^{s}\right)$. In all other cases, let us take $x^{s}=\hat{x}^{s}$. One should note that $x^{s} \in B^{i}\left(p^{s}, \delta^{s}\right)$.

If $p^{s} \rightarrow p^{s_{0}}$, then $\left\langle p^{s}, \hat{x}^{s}-\omega^{i}\right\rangle \rightarrow\left\langle p^{s 0}, x^{s 0}-\omega^{i}\right\rangle=0$. Hence, $x^{s} \rightarrow x^{0}$.

$$
\operatorname{But}\left\langle p^{s}, \bar{x}-\omega^{i}\right\rangle \rightarrow\left\langle p^{s 0}, \bar{x}-\omega^{i}\right\rangle<0
$$
thus, $\left\langle p^{s}, x^{s}-\omega^{i}\right\rangle=0$ implies that $\lambda^{s} \rightarrow 1, x^{s} \rightarrow x^{0}$.

Hence, $F^{i}(p, \delta)$ is lower hemicontinuous QED.

Let us state

Lemma 2. Let the assumptions of Lemma 1 hold, $I=\{1\}$, and $\varphi(p)$ be the aggregate demand function. Then sets $\varphi(p)$ are non-empty, convex, and closed.

Before proving Lemma 2, let us recollect some facts. Conventional proofs of existence of an equilibrium require the preference relation to be both monotonic and convex (see e.g. [12-14]). Let us generalize these constraints [11] by requiring the preference relation to be: 
$\alpha)$ monotonic: $x+z \succeq x$ for any $x \in R_{+}^{l}, \quad z \in K$, and there is $N>0$ such that $x+z \succeq x$ holds for any $x, z \in R_{+}^{l},\|z\| \geq N$; and

$\beta$ ) convex in directional cone $T:$ for all $\lambda \in[0,1]$ and all $x \in R_{+}^{l}$,

$y \succeq x, z \succeq x, y-z \in T$ results in $\lambda y+(1-\lambda) z \succeq x$.

Here, $K \subset R_{+}^{l}$ is any nondegenerate cone containing a point with unit coordinates, and cone $T$ is the closure of set $R^{l} \backslash\left( \pm R_{+}^{l}\right)$.

The authors require preferences to be not only convex but strictly convex in directional cone $T$. Let us define such preferences as follows:

$\left.\beta^{\prime}\right)$ A preference relation is strictly convex in directional cone $\sum_{k} p_{k} x_{k}^{i 1}=T$, if for all $\lambda \in[0,1]$ and all $x \in R_{+}^{l}$, $y \succeq x, y \neq x, y-x \in T$ results in $\lambda y+(1-\lambda) x \succ x$.

Just as merely convex preferences, a preference that is strictly convex in directional cone $T$ is convex in the same cone. Indeed, let $y \succeq x$ and $z \succeq x, y-z \in T$. If $y \neq z, y \succeq z$ or $z \succeq y$ (since the preference relation is complete by definition). For definiteness, let us assume that $y \succeq z$. Replacing $x$ with $z$ in (2), one gets $\lambda y+(1-\lambda) z \succ z$. Hence, as the preference is transitive, $\lambda y+(1-\lambda) z \succ x$, i.e., (1) holds. The other cases are trivial.

As regards the requirements $\alpha$ ) and $\beta$ ), imposed on preference relations, one can note the following. Compared to the usual interpretation of monotonicity (see, e.g., [15]), where vector $z$ can be an arbitrary vector from $R_{+}^{l}$, the requirement $\alpha$ ) allows situations where, for example, a consumer without a specific quantity of a particular good cannot accept an increase in the quantity of some other product. With regard to the second part of the requirement $\alpha$ ), it is clear that large quantities of even a single good from a set of goods must be dynamically useful to the consumer by virtue of the good's market prospects.

To better understand what it means for a preference relation to be convex in directional cone $T$, let us assume that in (1), $\lambda=0,5$ and $y$ is equivalent to $x$. Then a distribution participant indifferent to $x$ and $y$ will prefer their average over each of them (the preference is strict if its convexity is strict). This happens if $y-x \in T$. A particular case is when $y-x \in T$ and $x_{k}=0, y_{r}=0$, but $x_{r} \neq 0, y_{k} \neq 0$. Then the $k-$ th and the $r$-th coordinates of vector $(x+y) / 2$ are no longer zero. It means that distribution $(x+y) / 2$ gives both good $k$ and good $r$ to the consumer, while distributions $x$ and $y$ give only one of those. Saturation is at work here: Starting with a certain level, further consuming just one kind of good becomes less and less attractive. Requiring this phenomenon to only hold for vectors $x, y, y-x \in T$ means that one can only mix substantially opposite sets. If, for example, $x, y$ are sufficiently similar, and the consumer is indifferent to both, then the consumer will not experience the need for $(x+y) / 2$, as that vector will have no obvious advantage over $x$ and $y$. But in this case, $x-y \notin T$. Here, one understands the similarity of vectors $x$ and $y$ in terms of their collinearity, that is that the relations of the respective coordinates of vectors $x$ and $y$ are close to each other, so that $x-y \notin T$.

The convexity in directional cone $T$ becomes evident when one considers the notion of indifference surfaces. As one remembers, two distributions $x$ and $y$ are said to belong to the same indifference surface if $y$ is equivalent to $x$. A preference relation is being convex in directional cone $T$, which means that any indifference surface confines an area that is convex in directional cone $T$. That preference relation's being strictly convex means that none of these surfaces contain a line segment parallel to some vector from $T$.

One can describe a preference relation using utility function $u(x)$. It has been proven [9] that each continuous preference relation has a continuous utility function representing this relation (both must be defined on convex sets). The class of functions that define preference relations that are convex in directional cone $T$ has been established in [21] by means of the usual quasiconcavity approach. It has been proven that a preference relation is convex in directional cone $T$ if all utility functions representing it are quasiconcave in directional cone $T$.

Function $u(x)$ is said to be quasiconcave in directional cone $T$ if for any real $a$, the set

$$
S_{a}=\left\{z \in R_{+}^{l} \mid u(\mathrm{z}) \geq a\right\}
$$

is convex in directional cone $T$. Obviously, by a set convex in directional cone $T$ let us mean a set such that, for any two of its points $\mathrm{z}, y$ such that $y-z \in T$, the vector $\lambda y+(1-\lambda) z$ belongs to that set for any $\lambda \in[0,1][22]$.

Now let us go back to Lemma 2.

Proof of Lemma 2. Let us consider the $i$ - -th preference. Let us take set $B^{i}(p, \delta)$ and consider correspondence $M^{i}(p)$ that selects the most preferred elements for preference $\succeq_{i}$ on set $B^{i}(p, \delta)$. By virtue of the requirements imposed on the preference relation, the utility function $u_{i}(x)$ will be 
continuous for any $i$. Hence it reaches its maximum value on the compact set $B^{i}(p, \delta)$ at some point $x^{i}$. This point belongs to set $B^{i}(p, \delta)$. Thus, $M^{i}(p) \neq \varnothing$. Let $x^{i} \in M^{i}(p)$. Let us assume that $\left\langle p, x^{i}\right\rangle\left\langle\left\langle p, \omega^{i}\right\rangle\right.$ and demonstrate that it leads to a contradiction. Since this is a strict inequality, it will still hold if we increase the first component $x^{i}$ by a sufficiently small increment $\varepsilon>0$

$$
p_{1}\left(x_{1}^{i}+\varepsilon\right)+\sum_{k=2}^{l} p_{k} x_{k}^{i}<\sum_{k=1}^{l} p_{k} \omega_{k}^{i} .
$$

Let $z^{i}=\left(x_{1}^{i}+\varepsilon, x_{2}^{i}, \ldots, x_{l}^{i}\right)$. Clearly, vector $z^{i}$ is not equal to $x^{i}$ and is preferred over $x^{i}$. The latter holds since $I=\{1\}$.

But vector $x^{i}-$ is preferred over all other vectors by definition. Hence there is contradiction.

Now let us prove that $M^{i}(p)$ is convex. If $x^{i 1}, x^{i 2} \in M^{i}(p)$, then $\sum_{k} p_{k} x_{k}^{i 1}==\sum_{k} p_{k} x_{k}^{i 2}$ by virtue of the previous proof. But then $x^{i 1}-x^{i 2} \in T$, and, because the preference is $T$-convex, set $M^{i}(p)$ is convex. Thus, by following a well-known reasoning [see e.g. 12], one comes to assert Lemma 2. Lemma 2 is thus proven.

Let us note that if the preference matches the condition $\alpha$ ) for the cone $K \subset \beta^{i}(\delta)$ and is strictly convex in directional cone $T$, then set $M^{i}(p)$ consists of a single point. This holds even if no conditions have been imposed on the set $I$.

Indeed, set $M^{i}(p)$ is not empty by virtue of the requirements imposed on the preference relation. Let us assume that there are two points $x^{i}, z^{i} \in M^{i}(p)$ such that $x^{i} \succeq y, z^{i} \succeq y$ for any $y \in \alpha^{i}(p)$. By first assuming $y=x^{i}$ and then $y=z^{i}$ in these relations, one gets $x^{i} \sim z^{i}$. Now let us make sure that $x^{i}-z^{i} \in T$. To do this, it suffices to test that $\left\langle p, x^{i}\right\rangle=\left\langle p, z^{i}\right\rangle=\left\langle p, \omega^{i}\right\rangle$. If, for example, $\left\langle p, x^{i}\right\rangle\left\langle\left\langle p, \omega^{i}\right\rangle\right.$, one proceeds as follows. Let us take two vectors $b, c \in K, b \neq c$ such that $\left\langle p, \mathrm{~b}+x^{i}\right\rangle=\left\langle p, \mathrm{c}+x^{i}\right\rangle=\left\langle p, \omega^{i}\right\rangle$. Let $d_{1}=b+x^{i}, d_{2}=c+x^{i}$. Through their definition and the monotonicity of preference relation $\succeq_{i},\left\langle p, d_{1}\right\rangle=\left\langle p, d_{2}\right\rangle$; hence, $d_{1}-d_{2} \in T$. Since preference relation $\succeq_{i}$ is strictly convex in directional cone $T,\left(d_{1}+d_{2}\right) / 2 \succ_{i} x^{i}$. The latter contradicts the maximality of $x^{i}$, hence assumption $\left\langle p, x^{i}\right\rangle\left\langle\left\langle p, \omega^{i}\right\rangle\right.$ is false. Thus, set $M^{i}(p)$ consists of a single point.

The following theorem holds:
Theorem. Let $E=\left\{\left(X^{i}, \succeq_{i}, \omega^{i}\right), p\right\}$ satisfy Assumptions 1, 2 with index set $\mathrm{I}=\{1\}$ and 3 ; where $P$ is defined for some $\mu, \mu \in(0 ; 1)$ as $P=\left\{p \in R_{+}^{l} \mid p_{1}=1, \rho\left(p, p^{0}\right) \leq \sqrt{2(1-\mu)}\right\}$.

Then such exchange economy has a rationed equilibrium.

Proof. Let $\bar{p}$ be a vector where $\left\langle\bar{p}, p^{0}\right\rangle=\mu \cdot\|\bar{p}\| \cdot\left\|p^{0}\right\|$ and $\beta$ the angle between $p^{0}$ and $\bar{p}+\omega^{i}$. Let $\tau=\left\langle p^{0}, \bar{p}+\omega^{i}\right\rangle /\left(\left\|p^{0}\right\| \cdot\left\|\bar{p}+\omega^{i}\right\|\right)$.

Let us consider all vectors the angle between which and $p^{0}$ is not greater than $\beta$.

We get a compact, convex set $Q=\left\{q \in R_{+}^{l} \mid \rho\left(q, p^{0}\right) \leq \sqrt{2(1-\tau)}\right\}$.

For every $q \in Q$, let us define the price system $p(q)$ and the rationing scheme $\delta(q)$ by :

$$
(D):\left\{\begin{array}{c}
p(q)=\left\{\begin{array}{l}
q, \text { if } \rho\left(q, p^{0}\right) \leq \sqrt{2(1-\mu)}, \\
p, \text { if } \rho\left(q, p^{0}\right)=\sqrt{2(1-\mu)},
\end{array}\right. \\
\delta(q)=\left\{\begin{array}{c}
\beta-\alpha, \text { if } \rho\left(q, p^{0}\right) \leq \sqrt{2(1-\tau)}, \\
\beta-\gamma, \text { if } \rho\left(q, p^{0}\right)>\sqrt{2(1-\tau)}, \gamma<\beta,
\end{array}\right.
\end{array}\right.
$$

where $\gamma$ is the angle between $q$ and $p^{0}$.

It is obvious that for all $q \in Q$ : $\left(p(q), p^{0}\right) \geq \mu\|p(q)\|\left\|p^{0}\right\|, \quad\left(x, \omega^{i}\right) \geq \delta(q)\|x\|\left\|\omega^{i}\right\|, \quad$ and $F^{i}(p(q), \delta(q))=F^{i}(q)$ is a both upper and lower hemicontinuous vector-valued function of $q$ for all $q \in Q$.

Let $x^{i} \in B^{i}(q)$, then it follows from $(D)$ that $\left(q+x^{i}-\omega^{i}\right)=\left(q+z^{i}\right) \in Q$.

Let us consider $p=p(q), \delta=\delta(q)$ for some $q \in Q$ such that $q_{1}=1$. Let

$$
Q_{1}=\left\{q \in Q \mid \mathrm{q}_{1}=1\right\}, 1 q=\left(q_{2}, \ldots, q_{l}\right), Q^{\prime}=\left\{1 q \in R^{l-1} \mid(1,1 q) \in Q_{1}\right\}
$$

Let the correspondence $M^{i}(q)$ select the maximal elements for the preference $\succeq_{i}$ across the whole budget set $B^{i}(q)$. From the lemma proven above, $F^{i}(q)$ is both upper and lower hemicontinuous for each $[p(q), \delta(q)]$ and the correspondence $M^{i}(q): Q_{1} \rightarrow X^{i}$ is upper hemicontinuouson $Q_{1}$. Because the good 1 is always desired, $\left(p(q),\left(x^{i}-\omega^{i}\right)\right)=0$ for all $x^{i}$ from the output set of correspondence $M^{i}(q)$. Because $B^{i}(q)$ is compact and $\succeq_{i}$ is 
continuous and convex in directional cone $R_{+}$, then $M^{i}(q)$ is non-empty and convex [15].

Let us define excess demand as $\sigma(q)=\left\{z \in R^{l} \mid z+\sum \omega^{i} \in \sum M^{i}(q)\right\}$.

Let $1 z=\left(z_{2}, \ldots, \mathrm{z}_{l}\right)$ and one is to define a correspondence $\theta: Q^{\prime} \rightarrow Q^{\prime}$ by:

$$
\theta(1 q)=\left\{1 q^{\prime} \in R^{l-1} \mid 1 q^{\prime}=1 q+1 z / m, z \in \sigma(q)\right\} .
$$

$\theta(1 q)$ is convex, because $\sigma(q)$ is convex. $\theta$ is upper hemicontinuous, because $\sigma$ is upper hemicontinuous. $\theta(1 q) \subset Q^{\prime}$, because for all $x^{i} \in B^{i}(q)$, and $Q^{\prime}$ is convex. Also, $Q^{\prime} \neq \varnothing$ is compact.

All the conditions of the Kakutani theorem (see, e.g., [13, 23]) are satisfied, and $\theta$ has a fixed point. There are $q^{*} \in Q_{1}$ and $z^{*} \in \sigma\left(q^{*}\right)$ with $1 z^{*}=0$. Because $\left(p(q), \mathrm{z}^{i}\right)=0$ for all $z^{i} \in \sigma^{i}(q), 1 z^{*}=0$ implies $z^{*}=0$. There are $\left\{x^{i *}\right\}$, $p^{*}=p\left(q^{*}\right), \delta^{*}=\delta\left(q^{*}\right)$, such that conditions (a) and (b) in the equilibrium definition are satisfied for $E$. System $(D)$ was defined so as to also satisfy condition (c). Hence a rationed equilibrium exists. QED.

In conclusion, let us note that that exchange economies where resources and prices for goods belong to some cones have an optimal distribution. This distribution is characterized by representing the best set of goods that a consumer can obtain under given resource and price constraints.

\section{References}

[1] L.V. Kantorovich, P.K. Katyshev, A.Ya. Kiruta, V.M. Polterovich, "Some research directions in mathematical economics", Progress in Science and Technology, Current Problems in Mathematics, V.19, VINITI, Moscow, pp. 3-21, 1982.

[2] V.M. Polterovich, "Optimal distribution of goods under non-equilibrium prices", Economics and Mathematical methods, V. 16, Issue 4. pp. 95104, 1980.

[3] M.I. Levin, V.L. Makarov, A.M. Rubinov, "Mathematical models of economic interaction, Nauka, Moscow, Series Physical and Mathematical Literature, 1993.

[4] E.M. Bravermann, M.I. Levin, "Non-equilibrium models of economic systems", Nauka, Moscow, 1981.
[5] E.M. Bravermann, "Production models with non-equilubrium prices", Economics and Mathematical Methods, V.8, Issue 2, pp.71-79, 1979.

[6] Dzh.KH. Dreze, "Sushchestvovaniye ravnovesnogo obmena pri tsenovoy rigidnosti”, Mezhdunarodnyy Economic Review, T. 16, № 2, pp. 301-320, 1975.

[7] Dzh. Van Khorn, Dzhon M. Vakhovich. Osnovy finansovykh menedzherov, 13-ye izd., FT Prentice Hall, Kharlou, 2009.

[8] X. TSzyan, V. Chzhan, M. On, "A Study on the Selection Princnple of Industrial Clusters based on Industrial Integrated Design Case of Forestry", SHS Web of Conferences, ICMETM, vol. 17, pp.1-5, February 2015 [International Conference in Modern Economic Technology and Managment, https://doi.org/10.1051/shsconf/ 20151701001, 2015].

[9] S. Moiseyev, "Ratsionirovaniye kredita, ili kreditnyy payek dlya rossiyskoy ekonomiki”, Bankovskoye obozreniye, №4, pp.6, 2009.

[10] Liseychikova, R.T.Balakina, "Raising of credit portfolio as a credit risk management method", Bulletin of Omsk University, Series Economics, No 3, pp.173-179, 2009.

[11] M.A. Sevodin, O.I. Semenova, "On competetive equilibria in economic exchange growth models with price restrictions", Bulletin Inzhekona, Series Economics, Issue 6 (57), pp. 103-106, 2012.

[12] Kh. Nikaydo, Vypuklyye strukturyi ekonomicheskaya teoriya, Academic Press, New-York, 1968.

[13] Ivar Ekeland, Evolyutsiya matematiki, Izdaniya Germann, T. 23, Collection Méthodes, 1979.

[14] V.M. Polterovich, "Economic equilibrium and Economic Mechanism", USSR Academy of Sciences, Central Economic Mathematical Institute, Moscow: Nauka, 1990.

[15] Edmond Malinvaid, Lecons de theorie microeconomique, Dunod, Paris, 1977.

[16] L. McKenzie, "Demand theory without a utility index", Rev. Econ. Stud., V. 24 (3), No 65, pp. 185-189, 1956.

[17] R.J. Aumann, "Existence of competitive equilibria in markets with a continuum of traders", Econometrica, V. 34, pp. 1-17, 1966.

[18] A. Yamazaki, "An equilibrium existence theorem without convexity assumptions", Econometrica, V. 46, pp. 541-555, 1978.

[19] Ed. K. J. Arrow, M.D. Intrilligator, Handbook of mathematical economics, vol. 2, North-Holland, Amsterdam, 1982.

[20] Ed. K. J. Arrow, M.D. Intrilligator, Handbook of mathematical economics, vol. 3, North-Holland, Amsterdam, 1982.

[21] G. Debreu, Theory of Value: An Axiomatic Analysis of Economic Equilibrium, Yale University Press, New Haven and London, 1972 [1959].

[22] M.A. Sevodin, M.O. Gavrilova, On existence of equilibrium in exchange economics with one class of nonconvex consumers'preferences", Bulletin of Kazan University, Series Physical Mathematical Sciences, V.153,issue 1, pp.240-248, 2011.

[23] Jean-Pierre Aubin, L'analyse non lineaire et ses motivations economiques, Université de Paris - Dauphine, Ecole Polytechnique, Collection Mathématiques appliquées pour la maîtrise, 1984. 